\title{
Scaling Edge Computing through S-BVT and Pb/s Switching Devices in Large Dense Urban Metro Networks
}

\author{
G. Otero*, J. P. Fernández-Palacios**, M. Svaluto Moreolo***, D. Larrabeiti*, J. A. Hernández*, \\ J. M. Fabrega ${ }^{* * *}$, V. López ${ }^{* *}$, L. Nadal***, R. Martínez ${ }^{* * * *}$ \\ * Universidad Carlos III de Madrid, Spain \\ ** Telefónica de España, Spain \\ *** Centre Tecnológic de Telecomunicacions de Catalunya, Castelldefels, Spain. \\ e-mail: gaoterop@it.uc3m.es
}

\begin{abstract}
Dense metropolitan areas are responsible for the majority of the traffic growth in telecom operators. The focus of the EU H2020 PASSION project is to develop new photonic technologies for supporting agile metro networks and enabling capacities of $\mathrm{Tb} / \mathrm{s}$ per channel to deal with such traffic increment, with a particular focus on two components (1) the Sliceable Bandwidth Variable Transceivers (S-BVT) and (2) Pb/s optical switches. This paper analyses two strategies from the perspective of computation resource consumption: overflow over a paired samelevel DC and overflow over a centralized site. We present a methodology to compare both schemes and prove that a proper distribution of computing resources in the centralized overflow together with S-BVT high-speed transmission can outperform the costs of a distributed strategy, requiring fewer processors and much smaller DCs. Keywords: S-BVT, Metro Are Network, Software Defined Networking, Edge Computing, overflow traffic, PASSION project.
\end{abstract}

\section{INTRODUCTION}

There is a clear worldwide pattern in the increment of population around metropolitan areas. Such dense metropolitan areas are responsible for the majority of the traffic growth. Cloud-based services, video traffic, enterprise applications, and virtualization have led to a relentless rise the pressure in metropolitan areas, thus motivating the utilization of optical technologies to cope with such massive traffic increment. Combined with 5G mobility, such cloud services generate traffic flows exceeding typical current networks capacity.

However, scaling the deployment of edge computing to all Central Offices (COs) to support ultra-low latency applications is complex and costly. Dimensioning DCs to meet blocking probabilities as low as $10^{-6}[1]$ requires taking full advantage of state-of-the-art distributed computing capabilities over the MAN to keep a high CPU utilization. To this end, several DC interconnection strategies are feasible. However, a strict control of latency is required in order to achieve service response times within the limits imposed by the target applications. One way to reduce latency is by offloading edge computing traffic from IP and using direct optical channels to interconnect data centers according to the demand. The switching and multiplexing technology developed within EU project PASSION aims to enable such low-latency jitter-free inter-data center communication, among other applications. Since many MANs have edge-to-core distances below $40 \mathrm{Km}$ (i.e. $200 \mu s$ of one-way latency over optical fiber) the distribution of edge computation beyond the Central Office (CO) over other MAN DCs can be acceptable for most applications. This makes it possible to use several strategies.

Pairing same-level DCs (i.e. pair-wise strategy A in Figure 2), has three relevant advantages: (1) the latency for overflown traffic is not substantially larger than for locally served traffic; (2) the amount of optical channels is fewer in $A(N / 2)$ vs $(N)$ in $B$, where $N$ is the amount of COs; (3) the optical channels required to interconnect two DCs are also shorter in $A$ than in $B$. These advantages make, in principle, $A$ preferable to $B$. However, strategy A considers the provisioning of permanent physical infrastructure to cope with the overflow traffic. Conversely, the advent of dynamic Sliceable Bandwidth Variable Transceivers (S-BVT) can change this situation since dynamic temporal circuits can be provisioned for the overflown traffic over already existent links. The flex grid channels are set up by means of an SDN control plane yielding sub-second control of switches [2] and enabling end-to-end set up times in the order of units of seconds.

In this light, the focus of the EU H2020 PASSION project is to develop new photonic technologies for supporting agile metro networks and enabling capacities of $\mathrm{Tb} / \mathrm{s}$ per channel to deal with such traffic increment while keeping latency within strict low limits [3]. The new metro network infrastructure is defined in the project has two key components: (1) the Sliceable Bandwidth Variable Transceivers (S-BVT) and (2) Pb/s optical switches. PASSION effort is devoted to the development of the essential photonic building blocks, but at the same time is looking for a solution that fits within the network operator roadmap and targets at least a tenfold reduction in components energy consumption and footprint. Due to space and cost constraints the Central Office is envisaged to host a limited amount of resources and resort to other data centers within the latency budget to provide very low blocking probabilities. Providing an optical solution based on SDN programmability, an S-BVT can provide a dynamic multi-destination $\mathrm{Tb} / \mathrm{s}$ flows that can be adapted to deal with the computing demands overflown from the edge data centers are feasible by using a single transceiver per node. 
In this paper, we review the features of the latest generation S-BVTs and analyze this use case enabled by a fully programmable control plane. We present a methodology to model and compare both scenarios to prove that, a proper distribution of computing resources in the centralized overflow approach can improve the costs of a samelevel distributed strategy, requiring fewer processors and much smaller DCs in COs.

\section{SLICEABLE BANDWIDTH VARIABLE TRANSCEIVER (S-BVT)}

In order to address the flexibility and cost requirements of metro networks while providing high capacity, Sliceable Bandwidth Variable Transceivers are proposed as the multiplexing and switching technology to interconnect MAN nodes with maximum elasticity and simplicity. VCSEL (Vertical-Cavity Surface Emitting Laser) technology and dense photonic integration can be exploited to achieve a radical reduction of cost, power consumption and footprint.

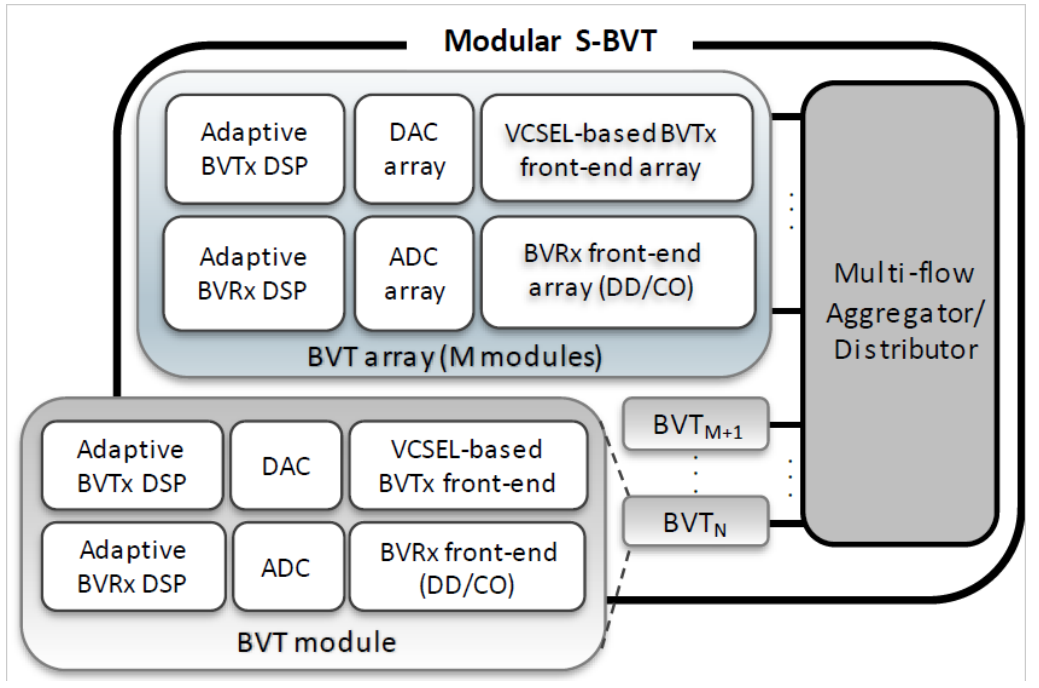

Figure 1. S-BVT modular architecture; in the inset: BVT adopting VCSEL-based $B V T x$ and alternative DD/CO BVRx.

As shown in Figure 1, the proposed S-BVT architecture is composed of an array of BVT modules, adopting VCSEL technology at the bandwidth variable transmitter (BVTx). The bandwidth variable receiver (BVRx) uses coherent $(\mathrm{CO})$ or direct detection (DD), trading ultimate performance for cost-effectiveness. The adaptive digital signal processing (DSP) allows a very fine spectral manipulation at the electrical subcarrier level, adopting multicarrier modulation (DMT or OFDM) with bit and power loading (BL/PL) algorithms. The multiple sliceable flows are aggregated/distributed at the S-BVT and/or the network node (with a spectrum selective switch).

The use of direct modulated (DM) VCSELs dramatically reduces the BVTx cost compared to external modulation or other DM lasers. Promising performance has been recently demonstrated in the long-wavelength range. A BVT module equipped with widely tunable $(70 \mathrm{~nm})$ VCSEL and DD has been demonstrated to support capacity above $30 \mathrm{~Gb} / \mathrm{s}$ in back-to-back and greater than $20 \mathrm{~Gb} / \mathrm{s}$ up to a 2-hop path of $185 \mathrm{~km}$, with flex grid spectral occupancy of $25 \mathrm{GHz}$ (DMT) and $12.5 \mathrm{GHz}$ (SSB-OFDM) [4]. Adopting larger bandwidth VCSELs and high-performance digital-to-analog and analog-to-digital converters (DAC/ADC), higher capacities can be achieved, as demonstrated for short-reach applications at $100 \mathrm{~Gb} / \mathrm{s}$ [5]. The achievable reach can be extended with CO-Rx. A scalable S-BVT architecture based on this approach can be implemented as modular pluggable units, each integrating multiple (M) VCSELs operating at different wavelengths at the BVTx array. Each element is suitably enabled/disabled for wavelength selection and adaptively loaded for BV adaptation. Dense photonic integration can be also adopted at the DD or CO BVRx array. The proposed modular S-BVT enables to support terabit capacities in a scalable and cost-effective fashion, targeting low power consumption and footprint. Furthermore, polarization and space dimensions can also be exploited. 

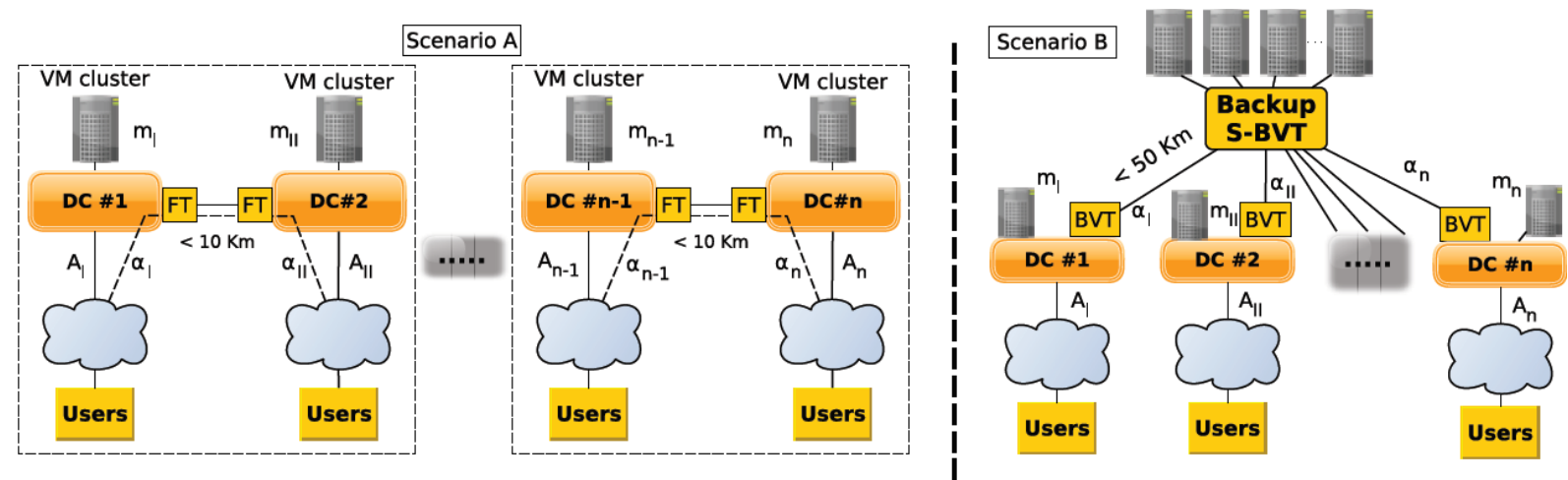

Figure 2. Overflow strategies: Pairwise (A) and Centralised (B).

\section{TRAFFIC AND COST ANALYSIS: NUMERICAL EXAMPLE}

Consider the two approaches for the target use case depicted in Figure 2. Strategy A represents a group of $n$ DCs located at $n$ metropolitan districts (approximately 200000 inhabitants). These implement a pair-wise protection system where both can send overflow traffic to one another. Strategy B shows a centralised backup system in which a S-BVT copes with the overflow traffic from all the local DCs.

Strategy A: consider that $1 \%$ of the population (2000 inhabitants) are subscribed to a service that is active 4 times a week per user, e.g., immersive live sports streaming, generating $2000 \cdot 4=8000$. Assuming exponentially distributed service times with a mean of 60 mins, the total offered traffic to each local DC is $A_{i}=47.619$ Erlangs for $i=\mathrm{I}, \mathrm{II}, \ldots, \mathrm{n}$. Considering Poissonian arrival times, the $i$-th local DC's blocking probability is given by the Erlang B expression $B_{i}=E\left(m_{i}, A_{\text {Total }}\right)$, where $m_{i}$ is the maximum number of available resources, e.g., virtual machines or processing units, at the local DC $i$, and $A_{\text {total }}$ is the aggregated offered traffic to each DC. This is the combination of the city users' traffic $\left(A_{i}\right)$ and the overflow traffic coming from the paired fixed transceiver (FT) of a neighbor city $\left(\alpha_{j}\right)$ (see Figure 2, left). Note that $A_{i}$ is poissonian while $\alpha_{j}$ follows an interrupted Poisson Process (IPP) and the blocking probability experienced by the aggregated traffic is greater than it would be if a Poissonian source was assumed. Consequently, we shall use the Fredericks-Hayward approximation to characterize the aggregated traffic and scale it by its peakedness factor. Let $r$ be the peakedness factor of one blocked arrival process, defined as the variance $v$ to mean $\alpha$ ratio of the occupancy: $r=\frac{v}{\alpha}=\frac{\sum_{i} \nu_{i}}{\sum_{i} \alpha_{i}}$, where $\alpha_{i}$ and $v_{i}$ are the amount and variance of the overflow traffic, respectively. Then, the blocking probability for each local DC follows

$$
B_{\text {LocalCDN }} \approx E\left(\frac{m_{i}}{r}, \frac{A_{\text {Total }}}{r}\right)
$$

Accordingly, the variance of each blocked flow can be computed via the Riordan's formula:

$$
v_{i}=\alpha_{i} \cdot\left(1-\alpha_{i}+\frac{A_{i}}{m_{i}+1-A_{i}}\right)
$$

Focusing on DC pair 1 - 2 in Scenario A Figure 2, we know that $A_{I, T o t a l}=A_{I}+\alpha_{I I}$. Also:

$$
\alpha_{I I}=B_{A_{I I}} \cdot A_{I I, \text { total }}=B_{A_{I I}} \cdot\left(A_{I I}+\alpha_{I}\right)
$$

Targeting a total blocking probability $B_{\text {total }}=10^{-6}$ (considering that we want a similar service availability as a tier 4 DC) and assuming that the overflow probability is equally distributed between the members of the pair, we get $B_{A_{I}}=B_{A_{I I}}=\sqrt{B_{\text {Total }}}=10^{-3}$. Substituting in Eq. (3) the target blocking probability and assuming $A_{I}=A_{I I}=$ 47.619 Erlangs, the overflow traffic is $\alpha_{I I}=\alpha_{I}=0.00477$ Erlangs. Therefore, the total offered traffic to each $\mathrm{DC}$ is $A_{I, \text { Total }}=A_{I I, \text { Total }}=47.6667$ Erlangs and the blocking probability is

$$
1 \cdot 10^{-3}=E\left(\frac{m_{i}}{r}, \frac{47.6667}{r}\right)
$$


which is a two-variable equation in $r$ and the number of resources $m_{i}$. Solving via bisection method, we obtain a peakedness factor of $r=1.0021$ and a number of resources at each local DC of $m_{i}=70$.

Strategy B: blocked traffic from the local DCs is steered towards the backup DC located at the MAN core (see Figure 2 right). We target the same overall blocking probability, i.e., $B_{\text {Total }}=10^{-6}$ but now it is distributed in a different way. Since in this case we have a backup infrastructure, we relax the blocking probability at the local DCs to be $B_{\text {LocalCDN }}=\sqrt[3]{B_{\text {Total }}}=10^{-2}$. Assume that the backup DC driven by the S-BVT is hopefully more reliable than the local DCs, achieving a blocking probability of $B_{\text {Backup }}=\frac{B_{\text {Total }}}{B_{\text {LocalCDN }}}=10^{-4}$.

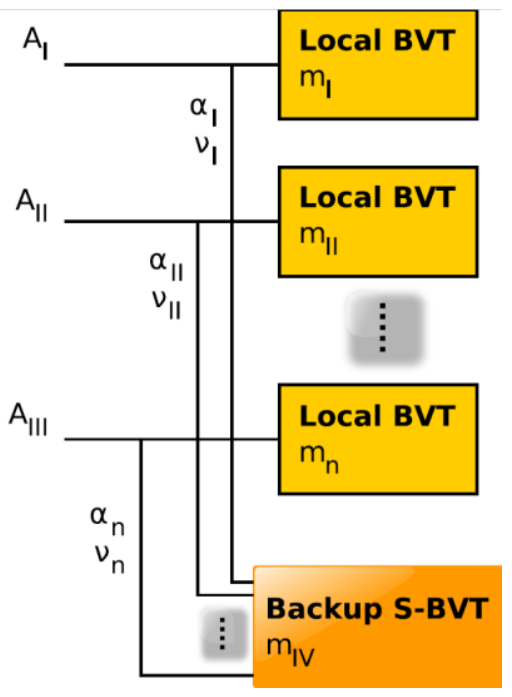

Figure 3. Traffic overflow in Scenario B.

Since now the only offered traffic to each local DC is the poissonian traffic, the blocking probability is: $B_{\text {LocalCDN }}=10^{-2}=E\left(m_{i}, 47.619\right) \leftrightarrow m_{i} \geq 61$. That is, we need only 61 resources to guarantee the same blocking probability as in Scenario A. The intensity of the overflow traffic, i.e., the blocked traffic at each local DC can be expressed as $\alpha_{i}=B_{i} \cdot A_{i}=0.4762$. Assuming a number of cities of $N_{C}=30$, the total overflow traffic is given by $A_{\text {Overflown }}=N_{C} \cdot \alpha_{i}=14.286$ Erlangs. Since all of the overflow traffic is non poissonian, we shall apply again Eq. (1) and (2) so as to find the number of resources needed to achieve the target overall blocking probability. After computing the peakedness factor as $r=\frac{N_{C} \cdot v_{i}}{N_{C} \cdot \alpha_{i}}=3.7289$, the blocking probability is

$$
B_{\text {Backup }, S B V T}=E\left(\frac{m_{S-B V T}}{3.7289}, \frac{14.286}{3.7289}\right)=10^{-4}
$$

yields $m_{S-B V T}=53$. We now compare the cost of both approaches for the same offered traffic to each local DC and the same overall blocking probability. Let $C_{\text {Local }}$ and $C_{\text {Core }}$ be the cost of a resource in a local DC and in the core DC driven by the S-BVT, respectively. Then, the total cost of each scenario in terms of computational resources are

$$
\begin{gathered}
\operatorname{Cost}_{A}=C_{\text {Local }} \cdot N_{C} \cdot m_{\text {iA }}=C_{\text {Local }} \cdot 2100 \quad(6) \\
\operatorname{Cost}_{B}=C_{\text {Local }} \cdot N_{C} \cdot m_{i B}+C_{\text {Core }} \cdot m_{S-B V T}=C_{\text {Local }} \cdot 1830+C_{\text {Core }} \cdot 53
\end{gathered}
$$

Figure 5 plots the total cost of both scenarios, for different target blocking probabilities, assuming a normalized cost of resources $C_{\text {Core }}=C_{\text {Local }}=1$. Additionally, we show the relationship between the costs of both scenarios as: $\left(\operatorname{Cost}_{A}-\operatorname{Cost}_{B}\right) / \operatorname{Cost}_{A}$ (see right axis green line with circles). Continuing our numerical example, total saving percentage for our target blocking probability is $8 \%$. It is important to note that the conclusion drawn is only true for certain values of the total blocking probability. Close inspection of Figure 5 reveals that Scenario B is better than Scenario A in terms of cost, for total blocking probabilities below $12.7 \cdot 10^{-3}$. Conversely, Scenario A remains better for blocking probabilities above that threshold. 


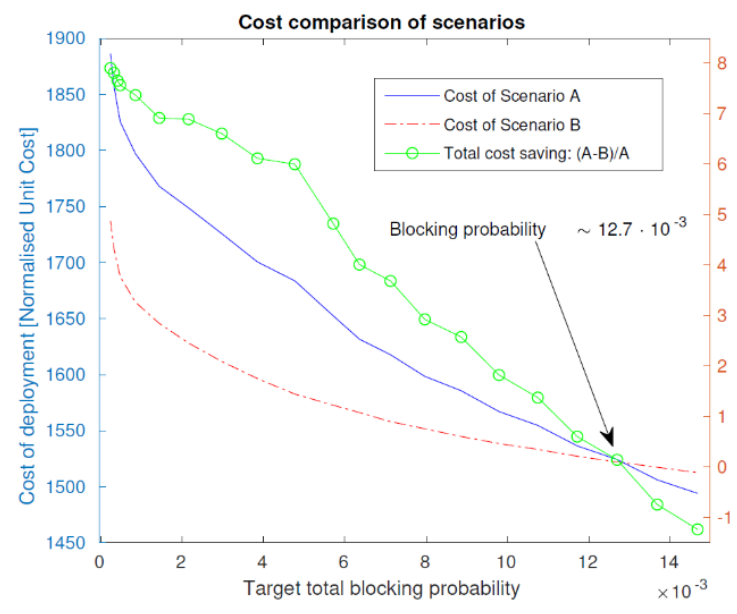

Figure 5. Cost comparison of scenarios.

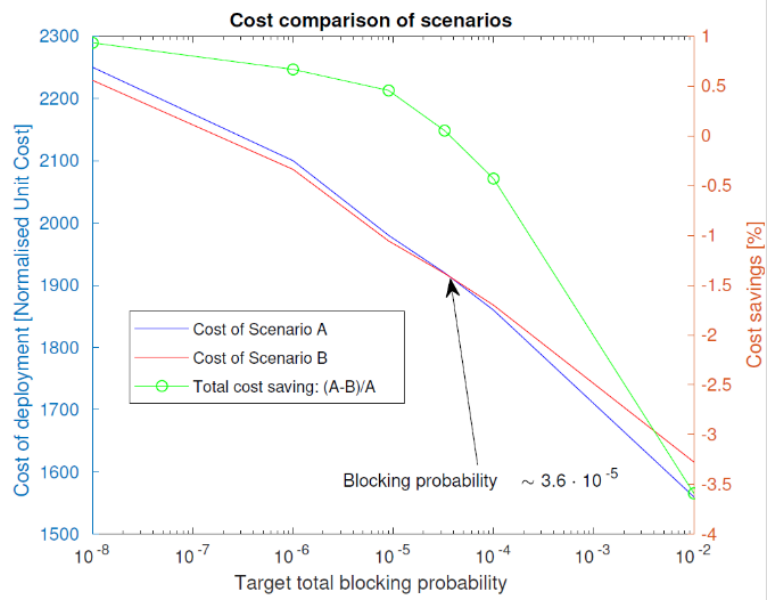

Figure 4. Blocking probability influence.

Finally, Figure 4 shows that both the gap between the cost of both approaches and the threshold that makes Scenario A or B a better choice are hugely dependent on how the blocking probabilities are divided between tiers. While Figure 5 represents the case in which the backup DC has a lower blocking probability, Figure 4 shows the behaviour of the system when $B_{\text {LocalCDN }}=B_{\text {Backup }}=\sqrt{B_{\text {Total }}}$. In this case, the achieved savings with Scenario $\mathrm{B}$ are never above $1 \%$.

\section{CONCLUSIONS}

The SDN-based dynamic multi-destination Tb/s capability of EU PASSION project S-BVTs enable different strategies to deal with the computing demands overflown from the edge data centers. In fact, they are all feasible by using a single transceiver per node and an automatically switched optical network. The margin for the distribution strategy to be adopted depends on the latency budget of the edge application. In this paper we analysed two strategies from the perspective of computation resource consumption when the delay budget permits to choose: overflow over a paired same-level DC and overflow over a centralised site. We present a methodology to compare both schemes and prove that a proper distribution of computing resources in the centralised overflow approach can reduce the IT infrastructure cost of a distributed strategy, requiring smaller DCs in COs. However, the condition for the saving is not straightforward and a careful analysis of the system to achieve a target blocking probability is required.

\section{ACKNOWLEDGEMENTS}

Telefonica and CTTC would like to acknowledge the support of EU project PASSION (grant no. 780326). UC3M would like to acknowledge the support of the Spanish project TEXEO (grant no. TEC2016-80339-R) and TAPIR-CM (grant no. P2018/TCS-4496).

\section{REFERENCES}

[1] R. Arno et al.: Reliability of Data Centers by Tier Classification, in IEEE Trans. Industry Applications, 48(2), 2012

[2] R. Martínez, et. al.: "Proof-of-Concept Validation of SDN-Controlled VCSEL-based S-BVTs in Flexi-Grid Optical Metro Networks", in proc. of OFC 2019

[3] EU PASSION Project: Photonic technologies for progrAmmable transmission and switching modular systems based on Scalable Spectrum/space aggregation for future agIle high capacity metrO Networks.

[4] M. Svaluto Moreolo et al.: Modular SDN-enabled S-BVT Adopting Widely Tunable MEMS VCSEL for Flexible-Elastic Optical Metro Networks, OFC 2018.

[5] C. Xie et al.: Single VCSEL 100-Gb/s short reach system using discrete multi-tone modulation and direct detection" OFC 2015.

[6] O. Gonzalez de Dios et al.: First Demonstration of Multi-vendor and Multi-domain EON with S-BVT and Control Interoperability over Pan-European Testbed, ECOC 2015.

[7] M. Svaluto Moreolo, et al.: Exploring the Potential of VCSEL Technology for Agile and High Capacity Optical Metro Networks, in Proc. ONDM 2018, 14-17 May 2018, Dublin (Ireland).

[8] G. O. Pérez, J. A. Hernández and D. Larrabeiti: Fronthaul network modeling and dimensioning meeting ultra-low latency requirements for 5G, in IEEE/OSA Journal of Optical Communications and Networking, vol. 10, no. 6, pp. 573-581, June 2018. 